\title{
TOTAL MEAN CURVATURE OF IMMERSED SURFACES IN $E^{m}$
}

\author{
BY \\ BANG-YEN CHEN $\left({ }^{1}\right)$
}

\begin{abstract}
Total mean curvature and value-distribution of mean curvature for certain pseudo-umbilical surfaces are studied.
\end{abstract}

1. Introduction. In the classical theory of surfaces in a euclidean $m$-space $E^{m}$, the two most important curvatures are the so-called Gauss curvature $G$ and the mean curvature $\alpha$. It is well known that the Gauss curvature is intrinsic. The integral of Gauss curvature gives the beautiful Gauss-Bonnet formula, which holds for orientable compact surfaces as well as nonorientable ones,

$$
\int_{M} G d V=2 \pi \chi(M)
$$

where $d V$ and $\chi(M)$ denote the volume element and Euler-Poincare characteristic of $M$. For the mean curvature of a compact surface $M$ in $E^{m}$ we have $[2,1]$ (see also [5]),

$$
\int_{M} \alpha^{2} d V \geqslant 4 \pi
$$

The equality holds when and only when $M$ is an ordinary 2-sphere in an affine 3-space. It is an interesting problem to improve inequality (1.2) for some special surfaces in $E^{m}$. In [2, III] the author obtains some results of this problem for surfaces in $E^{4}$. In this paper we shall study this problem for pseudo-umbilical surfaces in $E^{m}$ (for the definition of pseudo-umbilicity see §2). In particular, we shall prove the following:

THEOREM 1. Let $M$ be a compact pseudo-umbilical surface in $E^{m}$ with nonnegative Gauss curvature. If we have

$$
\int_{M} \alpha^{2} d V \leqslant(2+\pi) \pi,
$$

then $M$ is homeomorphic to a 2-sphere.

THEOREM 2. Let $M$ be a compact flat pseudo-umbilical surface in $E^{m}$.

Received by the editors February 7, 1975. 53B25.

AMS (MOS) subject classifications (1970). Primary 53A05, 53C40, 53C65; Secondary

Key words and phrases. Total mean curvature, pseudo-umbilical surfaces, Clifford torus, real projective plane, normal connection, Laplace's equation.

(1) Research partially supported by NSF Grant GP-36684. 
Then we have

$$
\int_{M} \alpha^{2} d V \geqslant 2 \pi^{2}
$$

The equality sign holds if and only if $M$ is a Clifford torus, i.e., $M$ is the product surface of two plane circles with the same radius.

2. Preliminaries. Let $x: M \rightarrow E^{m}$ be an isometrical immersion of a surface $M$ in an $m$-dimensional euclidean space $E^{m}$ and let $\nabla$ and $\nabla^{\prime}$ be the covariant differentiations of $M$ and $E^{m}$ respectively. Let $X$ and $Y$ be two tangent vector fields on $M$. Then the second fundamental form $h$ is given by

$$
\nabla_{X}^{\prime} Y=\nabla_{X} Y+h(X, Y) \text {. }
$$

It is well known that $h(X, Y)$ is a normal vector field on $M$ and is symmetric on $X$ and $Y$. Let $\xi$ be a normal vector field on $M$; we write

$$
\nabla_{X}^{\prime} \xi=-A_{\xi}(X)+D_{X} \xi
$$

where $-A_{\xi}(X)$ and $D_{X} \xi$ denote the tangential and normal components of $\nabla_{X}^{\prime} \xi$. Then we have

$$
\left\langle A_{\xi}(X), Y\right\rangle=\langle h(X, Y), \xi\rangle
$$

where $\langle$,$\rangle denotes the scalar product in E^{m}$. A normal vector field $\xi$ on $M$ is said to be parallel (in the normal bundle) if $D \xi=0$. The mean curvature vector $H$ is defined by

$$
H=1 / 2 \text { trace } h \text {. }
$$

The length of $H$, denoted by $\alpha$, is called the mean curvature of $M$. If the mean curvature vector $H$ is nowhere zero and the second fundamental form $h$ satisfies

$$
\langle h(X, Y), H\rangle=\lambda\langle X, Y\rangle,
$$

for all tangent vectors $X, Y$ on $M$, then $M$ is said to be pseudo-umbilical.

Let $R$ and $R^{N}$ be the curvature tensors associated with connections $\nabla$ and $D$, i.e., $R$ and $R^{N}$ are given respectively by

$$
R(X, Y)=\left[\nabla_{X}, \nabla_{Y}\right]-\nabla_{[X, Y]}
$$

and

$$
R^{N}(X, Y)=\left[D_{X}, D_{Y}\right]-D_{[X, Y]}
$$

For a surface $M$ in $R^{m}$, if $R$ vanishes identically, then $M$ is said to be flat. If $R^{N}$ vanishes identically, then $M$ is said to have flat normal connection. Let $e_{1}$ and $e_{2}$ be orthonormal vector fields tangent to $M$. Then the Gauss curvature $G$ of $M$ is a well-defined intrinsic function on $M$ given by 


$$
G=\left\langle R\left(e_{1}, e_{2}\right) e_{2}, e_{1}\right\rangle .
$$

The Gauss and Ricci equations are given respectively by

$$
\begin{aligned}
& \langle R(X, Y) Z, W\rangle=\langle h(X, W), h(Y, Z)\rangle-\langle h(X, Z), h(Y, W)\rangle, \\
& \left\langle R^{N}(X, Y) \xi, \eta\right\rangle=\left\langle h\left(A_{\xi}(Y), X\right), \eta\right\rangle-\left\langle h\left(A_{\xi}(X), Y\right), \eta\right\rangle,
\end{aligned}
$$

where $X, Y, Z, W$ are vector fields tangent to $M$ and $\xi, \eta$ are vector fields normal to $M$. For the second fundamental form $h$, we define the covariant derivative, denoted by $\bar{\nabla}_{X}$, to be

$$
\left(\bar{\nabla}_{X} h\right)(Y, Z)=D_{X}(h(Y, Z))-h\left(\nabla_{X} Y, Z\right)-h\left(Y, \nabla_{X} Z\right) .
$$

The Codazzi equation is given by

$$
\left(\bar{\nabla}_{X} h\right)(Y, Z)=\left(\bar{\nabla}_{Y} h\right)(X, Z) .
$$

Let $e_{1}, e_{2}, \xi_{3}, \ldots, \xi_{m}$ be orthonormal vector fields, defined along $M$, such that $e_{1}, e_{2}$ are tangent to $M$ and $\xi_{3}, \ldots, \xi_{m}$ are normal to $M$. For each $r=3, \ldots, m$, we simply denote $A_{\xi_{r}}$ by $A_{r}$. Let

$$
A_{r}=\left(h_{i j}^{r}\right)_{i j=1,2} \text {. }
$$

With respect to the basis $e_{1}, e_{2}$, we have $h_{12}^{r}=h_{21}^{r}$. From (2.4) and (2.6) we find

$$
\begin{gathered}
H=\frac{1}{2} \sum\left(h_{11}^{r}+h_{22}^{r}\right) \xi_{r}, \\
G=\sum\left(h_{11}^{r} h_{22}^{r}-h_{12}^{r} h_{12}^{r}\right) .
\end{gathered}
$$

3. Mean curvature of pseudo-umbilical surfaces with $R=R^{N}=0$. In this section we shall prove the following results for later use.

THEOREM 3. Let $M$ be a flat pseudo-umbilical surface in $E^{m}$ with flat normal connection. Then the mean curvature $\alpha$ satisfies the following Laplace's equation,

$$
\Delta \ln \alpha=0,
$$

where $\Delta$ denotes the Laplacian on $M$.

Proof. Since the normal connection of $M$ is flat, the equation of Ricci implies that

$$
\left[A_{r}, A_{s}\right]=0, \quad r, s=3, \ldots, m .
$$

Now, let $\xi_{3}, \ldots, \xi_{m}$ be chosen in the way that $H=\alpha \xi_{3}$. Then by the pseudoumbilicity of $M$ in $E^{m}$, we have 


$$
A_{3}=\left(\begin{array}{ll}
\alpha & 0 \\
0 & \alpha
\end{array}\right), \quad A_{r}=\left(\begin{array}{cc}
h_{11}^{r} & h_{12}^{r} \\
h_{12}^{r} & -h_{11}^{r}
\end{array}\right), \quad r=4, \ldots, m .
$$

By (3.1), we may choose orthonormal tangent vectors $e_{1}, e_{2}$ which diagonalize $A_{r}$ simultaneously. With respect to such frame $e_{1}, e_{2}, \xi_{3}, \ldots, \xi_{m}$, we have

$$
A_{3}=\left(\begin{array}{ll}
\alpha & 0 \\
0 & \alpha
\end{array}\right), \quad A_{r}=\left(\begin{array}{cc}
h_{11}^{r} & 0 \\
0 & -h_{11}^{r}
\end{array}\right), \quad r=4, \ldots, m .
$$

Now, by the flatness of $M$ and (2.11), we have

$$
\alpha^{2}=\sum_{r=4}^{m}\left(h_{11}^{r}\right)^{2} \text {. }
$$

For each $p \in M$, let $N_{p}$ be the vector space consisting of all normal vectors of $M$ in $E^{m}$ at $p$ which are perpendicular to the mean curvature vector $H$. On $N_{p}$ we define a linear mapping into the set of all symmetric matrices of order 2 by $\rho(\xi)=A_{\xi}$. Let $O_{p}$ denote the kernel of $\rho$. Then by (3.3) and (3.4) we see that $\operatorname{dim} O_{p}=m-4$. Hence, we may choose a frame field $e_{1}, e_{2}, \widetilde{\xi}_{3}, \widetilde{\xi}_{4}, \ldots, \widetilde{\xi}_{m}$ such that, with respect to this frame, we have

$$
A_{3}=\left(\begin{array}{ll}
\alpha & 0 \\
0 & \alpha
\end{array}\right), A_{4}=\left(\begin{array}{cc}
\alpha & 0 \\
0 & -\alpha
\end{array}\right), \quad A_{s}=0, s=5, \ldots, m .
$$

Since the normal connection is flat, there exist, at least locally, orthonormal normal vector fields $\bar{\xi}_{3}, \ldots, \bar{\xi}_{m}$ such that $\bar{\xi}_{3}, \ldots, \bar{\xi}_{m}$ are parallel (see $[1$, p. 99]), i.e.,

$$
D \bar{\xi}_{3}=\cdots=D \bar{\xi}_{m}=0
$$

We put

$$
\bar{\xi}_{r}=\sum_{s=3}^{m} a_{r s} \tilde{\xi}_{s}, \quad r=3, \ldots, m .
$$

Then $\left(a_{r s}\right)$ is an orthogonal matrix of order $m-2$.

Since $M$ is two dimensional and local study of $M$ is sufficient, we may assume that $M$ is covered by an isothermal coordinate $(x, y)$ such that the metric on $M$ has the form $d s^{2}=E\left(d x^{2}+d y^{2}\right)$. In the following, we shall denote the coordinate vector fields $\partial / \partial x$ and $\partial / \partial y$ by $X_{1}$ and $X_{2}$ respectively. We put

$$
L=h\left(X_{1}, X_{1}\right), \quad M=h\left(X_{1}, X_{2}\right), \quad N=h\left(X_{2}, X_{2}\right)
$$

and

$$
\nabla_{X_{j}} X_{i}=\sum_{k=1}^{2} \Gamma_{j i}^{k} X_{k}, \quad i, j=1,2
$$


Then we have

$$
\Gamma_{11}^{1}=\Gamma_{12}^{2}=-\Gamma_{22}^{1}=X_{1} E / 2 E, \quad \Gamma_{22}^{2}=\Gamma_{12}^{1}=-\Gamma_{11}^{2}=X_{2} E / 2 E .
$$

Therefore the Codazzi equation reduces to

$$
\begin{gathered}
D_{X_{2}} L-D_{X_{1}} M=\left(X_{2} E\right) H \\
D_{X_{2}} M-D_{X_{1}} N=-\left(X_{1} E\right) H
\end{gathered}
$$

Since $X_{1}$ and $X_{2}$ are orthonormal, we may define a function $\theta=\theta(x, y)$ by

$$
\begin{aligned}
& X_{1} \equiv \partial / \partial x=\cos \theta e_{1}+\sin \theta e_{2}, \\
& X_{2} \equiv \partial / \partial y=-\sin \theta e_{1}+\cos \theta e_{2} .
\end{aligned}
$$

Then with respect to the frame field $X_{1}, X_{2}, \bar{\xi}_{3}, \ldots, \bar{\xi}_{m}$, the second fundamental tensors are givèn by

$$
A_{r}=\left(\begin{array}{cc}
\alpha\left(a_{r 1}+a_{r 2} \cos 2 \theta\right) & -\alpha a_{r 2} \sin 2 \theta \\
-\alpha a_{r 2} \sin 2 \theta & \alpha\left(a_{r 1}-a_{r 2} \cos 2 \theta\right)
\end{array}\right) .
$$

Since $M$ is flat, we may assume that $\dot{E}=1$. Hence by (3.6) equations of Codazzi reduce to

$$
\begin{aligned}
& \frac{\partial}{\partial y}\left[\alpha\left(a_{r 1}+a_{r 2} \cos 2 \theta\right)\right]=-\frac{\partial}{\partial x}\left[\alpha a_{r 2} \sin 2 \theta\right], \\
& -\frac{\partial}{\partial y}\left[\alpha a_{r 2} \sin 2 \theta\right]=\frac{\partial}{\partial x}\left[\alpha\left(a_{r 1}-a_{r 2} \cos 2 \theta\right)\right] .
\end{aligned}
$$

Multiplying $a_{r 1}$ to (3.8) and summing over $r$, then, by the fact that $\left(a_{r s}\right) \in$ $O(m-2)$, we find

$$
\frac{\partial \ln \alpha}{\partial y}=\sum_{r=3}^{m}\left\{\left(\frac{\partial a_{r 1}}{\partial y}\right) a_{r 2} \cos 2 \theta+\left(\frac{\partial a_{r 1}}{\partial x}\right) a_{r 2} \sin 2 \theta\right\} .
$$

Similarly, multiplying $a_{r 1}$ to (3.9) and summing over $r$, we have

$$
\frac{\partial \ln \alpha}{\partial x}=\sum_{r=3}^{m}\left\{\left(\frac{\partial a_{r 1}}{\partial y}\right) a_{r 2} \sin 2 \theta-\left(\frac{\partial a_{r 1}}{\partial x}\right) a_{r 2} \cos 2 \theta\right\} \text {. }
$$

Multiplying $a_{r 2}$ to (3.8) and summing over $r$, we find

$$
\begin{aligned}
\sum a_{r 2}\left(\frac{\partial a_{r 1}}{\partial y}\right)+\left(\frac{\partial \ln \alpha}{\partial x}\right) & \sin 2 \theta+\left(\frac{\partial \ln \alpha}{\partial y}\right) \cos 2 \theta \\
= & 2 \frac{\partial \theta}{\partial y} \sin 2 \theta-2 \frac{\partial \theta}{\partial x} \cos 2 \theta
\end{aligned}
$$


Hence, by substituting (3.10) and (3.11) into this equation, we find

$$
\sum a_{r 2} \frac{\partial a_{r 1}}{\partial y}=\sin 2 \theta \frac{\partial \theta}{\partial y}-\cos 2 \theta \frac{\partial \theta}{\partial x} .
$$

Similarly, by multiplying $a_{r 2}$ to (3.9), summing over $r$, and by using (3.10) and (3.11), we get

$$
\sum a_{r 2} \frac{\partial a_{r 1}}{\partial x}=-\cos 2 \theta \frac{\partial \theta}{\partial y}-\sin 2 \theta \frac{\partial \theta}{\partial x}
$$

Substituting (3.12) and (3.13) into (3.10) and (3.11), we may find

$$
\frac{\partial \ln \alpha}{\partial x}=\frac{\partial \theta}{\partial y}, \quad \frac{\partial \ln \alpha}{\partial y}=-\frac{\partial \theta}{\partial x} .
$$

From this we get $\left(\partial^{2} / \partial x^{2}\right) \ln \alpha+\left(\partial^{2} / \partial y^{2}\right) \ln \alpha=0$. Since $E=1$, this implies that $\Delta \ln \alpha=0$. Q.E.D.

It shall be remarked that for a pseudo-umbilical surface in $E^{4}$, the normal connection is always flat.

As an application of Theorem 3, we have the following result concerning about the value-distribution of mean curvature $\alpha$.

THEOREM 4. Let $M$ be a complete flat pseudo-umbilical surface in $E^{m}$ with flat normal connection. Then we have either

(1) the mean curvature $\alpha$ of $M$ takes every value in $(0, \infty)$, or

(2) the mean curvature $\alpha$ of $M$ takes only one value in $(0, \infty)$.

If case (2) holds, $M$ is the product of two curves $C_{1}$ and $C_{2}$ where $C_{1}$ is a curve in $E^{n}$ for some $n ; 1<n<m$; and $C_{2}$ is a curve in $E^{m-n}$ so that the first curvatures of $C_{1}$ and $C_{2}$ are equal.

Proof. Since $M$ is flat and complete, $M$ is parabolic in the sense that there exists no nonconstant negative subharmonic function on $M$. Thus every subharmonic function on $M$ which is bounded from above on $M$ must be a constant function. By Theorem $3, \ln \alpha$ is a continuous harmonic function and so a sub. harmonic and also a superharmonic function on $M$. Hence, if $\alpha$ does not take every value in $(0, \infty)$, then $\alpha$ must be constant. This proves the first part of the theorem.

Now, suppose that case (2) holds. Then $\alpha$ is a nonzero constant. From (3.5) we see that with respect to the frame field $e_{1}, e_{2}, \xi_{3}^{\prime}, \xi_{4}^{\prime}, \xi_{5}^{\prime}, \ldots, \xi_{m}^{\prime}$, we have

$$
A_{3}=\left(\begin{array}{cc}
\sqrt{2} \alpha & 0 \\
0 & 0
\end{array}\right),\left(\begin{array}{cc}
0 & 0 \\
0 & \sqrt{2} \alpha
\end{array}\right), \quad A_{5}=\cdots=A_{m}
$$


where $\xi_{3}^{\prime}=\cos \theta \tilde{\xi}_{3}+\sin \theta \tilde{\xi}_{4}, \xi_{4}^{\prime}=\sin \theta \tilde{\xi}_{3}-\cos \theta \tilde{\xi}_{4}, \xi_{5}^{\prime}=\tilde{\xi}_{5}, \ldots, \xi_{m}^{\prime}=\tilde{\xi}_{m}$. From (3.15) and the equations of structure we may easily find that both the distributions $T_{i}=\left\{a e_{i}: a \in R\right\}, i=1,2$ are parallel. By the de Rham decomposition theorem we see that $M=C_{1} \times C_{2}$ where $C_{1}$ (respectively, $C_{2}$ ) is the maximal integral manifold of $T_{1}$ (resp. $T_{2}$ ). Moreover, (3.15) implies that $h\left(e_{1}, e_{2}\right)=0$. Hence, $M$ is the product of $C_{1}$ and $C_{2}$ such that $C_{1}$ is a curve in an affine $n$-space $E^{n}$ and $C_{2}$ is a curve in an affine $(m-n)$-space $E^{m-n}$ in $E^{m}$. Let $h^{i}$ be the second fundamental form of $C_{i}, i=1,2$. Then, (3.15) implies $h^{i}\left(e_{i}, e_{i}\right)=\sqrt{2} \alpha \xi_{i+2}^{\prime}, i=1,2$. Hence the first curvatures of $C_{1}$ and $C_{2}$ are equal. Q.E.D.

4. Proofs of Theorems 1 and 2. Let $M$ be a compact pseudo-umbilical surface in $E^{m}$. Let $e_{1}, e_{2}, \xi_{3}, \ldots, \xi_{m}$ be a local field of orthonormal frame defined along $M$ such that $e_{1}, e_{2}$ are tangent to $M$ and $\xi_{3}, \ldots, \xi_{m}$ normal to $M$. For a unit normal vector $\xi$ at $p \in M$, the Lipschitz-Killing curvature $K(p, \xi)$ is defined by $K(p, \xi)=\operatorname{det} A_{\xi}$. Let $\xi=\Sigma_{r=3}^{m} \cos \theta_{r} \xi_{r}$ and $A_{r}=\left(h_{i j}^{r}\right)$. Then we have

$$
K(p, \xi)=\left(\sum \cos \theta_{r} h_{11}^{r}\right)\left(\sum \cos \theta_{s} h_{22}^{s}\right)-\left(\sum \cos \theta_{t} h_{12}^{t}\right)^{2} .
$$

The right-hand side of this equation is a quadratic form of $\cos \theta_{3}, \ldots, \cos \theta_{m}$. Hence, by choosing a suitable local frame field $\bar{\xi}_{3}, \ldots, \bar{\xi}_{m}$, we may write

$$
K(p, \xi)=\sum \lambda_{r-2}(p) \cos ^{2} \theta_{r}
$$

where $\lambda_{1}, \ldots, \lambda_{m-2}$ are continuous functions defined on $M$ and satisfy the following relations: $\lambda_{1} \geqslant \lambda_{2} \geqslant \cdots \geqslant \lambda_{m-2}$. From (2.11) we find

$$
G=\lambda_{1}+\cdots+\lambda_{m-2} \text {. }
$$

Since $M$ is pseudo-umbilical, if we choose $\xi_{3}$ in the direction of the mean curvature vector, then (3.2) holds. From this we may easily see that $K(p, \xi)$ takes its maximal value at $\xi_{3}$. Hence we have

$$
\lambda_{1}=\alpha^{2}, \quad \lambda_{2} \leqslant 0, \ldots, \lambda_{m-2} \leqslant 0 .
$$

Now, let $S_{p}$ be the unit $(m-3)$-sphere of all unit normal vectors at $p \in M$ and $d \sigma$ the volume element of $S_{p}$. Then the total absolute curvature $K^{*}(p)$ at $p$ is given by

$$
K^{*}(p)=\int_{S_{p}}|K(p, \xi)| d \sigma
$$

and the total absolute curvature $T A(M)$ of $M$ in $E^{m}$ (in the sense of Chern-Lashof [3] ) is given by $T A(M)=\int_{M} K^{*}(p) d V$. Let $H_{i}(M ; F)$ be the $i$ th homology group 
of $M$ over a field $F$ and $\beta_{i}(M ; F)$ the dimension of $H_{i}(M ; F)$. Then we have [3]

$$
T A(M) \geqslant c_{m-1} \beta(M),
$$

where $\beta(M)=\max \left\{\Sigma_{i=0}^{2} \beta_{i}(M ; F): F\right.$ fields $\}$ and $c_{m-1}$ the area of unit $(m-1)$ sphere.

From (4.2) and (4.3) we find

$$
\begin{aligned}
|K(p, \xi)| & =\left|G \cos ^{2} \theta_{3}+\sum_{r=4}^{m} \lambda_{r-2}\left(\cos ^{2} \theta_{r}-\cos ^{2} \theta_{3}\right)\right| \\
& \leqslant|G| \cos ^{2} \theta_{3}-\sum_{r=4}^{m} \lambda_{r-2}\left|\cos ^{2} \theta_{r}-\cos ^{2} \theta_{3}\right| .
\end{aligned}
$$

This implies that

$$
K^{*}(p) \leqslant \frac{c_{m-1}}{2 \pi}|G|-\sum_{r=4}^{m} \lambda_{r-2} \int_{S_{p}}\left|\cos ^{2} \theta_{r}-\cos ^{2} \theta_{3}\right| d \sigma .
$$

On the other hand, since

$$
\int_{S_{p}}\left|\cos ^{2} \theta_{r}-\cos ^{2} \theta_{3}\right| d \sigma=2 c_{m-1} / \pi^{2}
$$

(4.2), (4.3) and (4.5) imply

$$
\alpha^{2} \geqslant\left(\pi^{2} / 2 c_{m-1}\right) K^{*}(p)+G(p)-(\pi / 4)|G(p)| .
$$

Case (1). $G=0$. In this case, (4.4) and (4.6) imply

$$
\int \alpha^{2} d V \geqslant\left(\pi^{2} / 2\right) \beta(M) \text {. }
$$

On the other hand, the flatness of $M$ implies that $M$ is either homeomorphic to a torus or a Klein bottle; in both cases, $\beta(M)=4$. Hence (4.7) implies inequality (1.4). Now, if the equality of (1.4) holds, then we have

$$
T A(M)=4 c_{m-1} .
$$

Moreover, the inequality in (4.5) is actually an equality for all $\left(\theta_{3}, \ldots, \theta_{m}\right)$ satisfying $\cos ^{2} \theta_{3}+\cos ^{2} \theta_{4}+\cdots+\cos ^{2} \theta_{m}=1$. Hence we have

$$
\lambda_{m-2}=-\alpha^{2}, \quad \lambda_{2}=\cdots=\lambda_{m-3}=0 .
$$

From this we see that $\left[A_{r}, A_{s}\right]=0$ for all $r, s=3, \ldots, m$. By using equation (2.7) of Ricci, we see that the normal connection of $M$ in $E^{m}$ is flat. Thus, Theorem 4 implies that $M=C_{1} \times C_{2}$, where $C_{1}$ and $C_{2}$ are two closed curves in $E^{n}$ and $E^{m-n}$, respectively, with the same first curvature for some $n, 1<n<m$. On the other hand by a result of Kuiper [4], we have 


$$
T A(M) / c_{m-1}=T A\left(C_{1}\right) T A\left(C_{2}\right) / c_{n-1} c_{m-n-1},
$$

where $T A\left(C_{i}\right)$ is the total absolute curvature of $C_{i}, i=1,2$. Since $T A\left(C_{1}\right) \geqslant$ $2 c_{n-1}$ and $T A\left(C_{2}\right) \geqslant 2 c_{m-n-1},(4.8)$ implies $T A\left(C_{1}\right)=2 c_{n-1}$ and $T A\left(C_{2}\right)=$ $2 c_{m-n-1}$. From these we know that both $C_{1}$ and $C_{2}$ are two plane circles with the same radius [3]. Thus $M$ is a Clifford torus. This proves Theorem 2.

Case (2). $G \geqslant 0$ and $G \neq 0$. In this case, $M$ is either homeomorphic to a sphere or a real projective plane. Now, suppose that $M$ is homeomorphic to a real projective plane. Then we have $\chi(M)=1$ and $\beta(M)=3$. Hence inequality (4.4) implies

$$
\int_{M} \alpha^{2} d V \geqslant \frac{3}{2} \pi^{2}+\left(1-\frac{\pi}{2}\right) \int_{M} G d V
$$

This, combining with Gauss-Bonnet's formula, gives

$$
\int_{M} \alpha^{2} d V \geqslant(2+\pi) \pi
$$

In the equality of (4.10) holds, then the inequality in (4.5) is actually an equality for all $\left(\theta_{3}, \ldots, \theta_{m}\right)$ satisfying $\cos ^{2} \theta_{3}+\cdots+\cos ^{2} \theta_{m}=1$. Hence, we have either $\lambda_{2}=\cdots=\lambda_{m-2}=0$ or $G=\lambda_{3}=\cdots=\lambda_{m-2}=0$ pointwise. Now, let $U=\{p \in M: G(p) \neq 0\}$. Then $U$ is a nonempty open subset of $M$. By the assumption of pseudo-umbilicity, $U$ is totally umbilical in $E^{m}$. Hence the Gauss curvature $G$ is positive constant on every component of $U$ (see [1, p. 49]). From this we know that $U$ is also a closed subset of $M$. Thus $U=M$ and $M$ is an ordinary 2-sphere in $E^{m}$. This is a contradiction. This proves Theorem 1. Q.E.D.

REMARK 1. The real projective plane can be immersed in $E^{5}$ as a pseudoumbilical surface with positive constant Gauss curvature and total mean curvature $6 \pi$.

\section{REFERENCES}

1. B.-Y. Chen, Geometry of submanifolds, Dekker, New York, 1973.

2. - On the total curvature of immersed manifolds. I, II, III, Amer. J. Math. 93 (1971), 148-162; ibid. 94 (1972), 799-809; ibid. 95 (1973), 636-642. MR 43 \#3971; 47 \#7660; 48 \#12438.

3. S. S. Chern and R. K. Lashof, On the total curvature of immersed manifolds. I, II, Amer. J. Math. 79 (1957), 306-318; Michigan Math. J. 4 (1958), 5-12. MR 18, 927; 20 \#4301.

4. N. H. Kuiper, Immersions with minimal total absolute curvature, Colloq. Géom. Diff. Globale (Bruxelles, 1958), Centre Belge Rech. Math., Louvain, 1959, pp. 75-88. MR 23 \#A608.

5. T. J. Willmore, Mean curvature of immersed surfaces, An. Sti. Univ. "A1. I Cuza" Iași. Seçt. I a Mat. 14 (1968), 99-103. MR 38 \#6496.

DEPARTMENT OF MATHEMATICS, MICHIGAN STATE UNIVERSITY, EAST LANSING, MICHIGAN 48824 